Communications in Physics, Vol.22, No. 2 (2012), pp. 111-124

\title{
MAGNETOHYDRODYNAMICS IN PRESENCE OF ELECTRIC AND MAGNETIC CHARGES
}

\author{
P. S. BISHT \\ Department of Physics, Kumaun University \\ S. S. J. Campus, Almora-263601 (Uttarakhand) India \\ and \\ Institute of Theoretical Physics, Chinese Academy of Sciences \\ P. O. Box 2735, Beijing 100080, P. R. China \\ PUSHPA AND O. P. S. NEGI \\ Department of Physics, Kumaun University \\ S. S. J. Campus, Almora-263601 (Uttarakhand) India
}

\begin{abstract}
Starting from the generalized electromagnetic field equations of dyons, the magnetohydrodynamics (MHD) of plasma for particles carrying simultaneously the electric and magnetic charges (namely dyons), has been reformulated in terms of the electromagnetic duality. Consequently, the frequency of dyonic plasma is obtained for real value of wave number $k$. In this case, only those generalized electromagnetic waves are allowed to pass, for which the usual frequency is greater than the plasma frequency (i.e. $\omega>\omega_{p}$ ). Accordingly, the Ohm's law has been re-described to derive the plasma oscillation equation, Magnetohydrodynamic wave equation and to calculate the energy of dyons in unique and consistent manner.
\end{abstract}

\section{INTRODUCTION}

Magneto hydrodynamics (MHD) is a branch of the science of the dynamics of matter moving in an electromagnetic field [1-3] and thus provides one of the most useful fluid models, focusing on the global properties of plasma. The field of MHD was initiated by Alfvén [4] with the idea that magnetic fields induces currents in a moving conductive fluid so that it creates forces and changes the magnetic field. The set of equations which describe MHD are a combination [1] of the Navier - Stokes equations of fluid dynamics and Maxwell's equations of electromagnetism. In a series of papers [5-10], we have undertaken the study of dual electrodynamics, superluminal electromagnetic fields, quaternionic formulation of dyons in isotropic homogeneous, chiral and inhomogeneous media and obtained the solutions for the classical problem of moving dyon in unique and consistent way. P. Olesen [11] has shown that dual strings could be a solutions of the magnetohydrodynamics equations in the limit of infinite conductivity. Yeh-Dryer [12] discussed the multi dimensional MHD solutions for fictious monopole, while paper Dellar [13] has taken the case of one dimensional MHD Riemann problem for magnetic monopole. The "magnetic scenario" for the quark gluon plasma has also been viewed by Shuryak [14] considering two plasma states one of which electrically charged particle and other is magnetically 
charged quasi particle. Coceal et. al. [15] derived consistently the duality invariant magnetohydrodynamics and their dyonic solutions. Keeping in view the recent interest on the potential importance of magnetic monopole and their possible role in MHD and plasma, in this paper, we have undertaken the study of magnetohydrodynamics (MHD) of plasma for particles carrying simultaneously the electric and magnetic charges (namely dyons). It is shown that the resultant system supports the electromagnetic duality of dyons. Consequently, the frequency of dyonic plasma has been obtained and it is emphasized that there is a different plasma frequency for each species depending on wave number $k$. For $k$ to be real, only those generalized electromagnetic waves are allowed to pass, for which the usual frequency is greater than the plasma frequency (i.e. $\omega>\omega_{p}$ ). It is also shown that the plasma frequency sets the lower cuts for the frequencies of electromagnetic radiation which can pass through a plasma. Accordingly the Ohm's law has been re-described to derive the plasma oscillation equation, the magnetohydrodynamic wave equation and to calculate the energy of dyons in unique and consistent manner.

\section{FIELDS ASSOCIATED WITH DYONS}

Postulating the existence of magnetic monopoles, the generalized Dirac Maxwell's (GDM) equations [16] are expressed in SI units $(c=\hbar=1)$ as

$$
\begin{aligned}
\vec{\nabla} \cdot \vec{E} & =\rho_{e} ; \\
\vec{\nabla} \cdot \vec{H} & =\rho_{g} ; \\
\vec{\nabla} \times \vec{E} & =-\frac{\partial \vec{H}}{\partial t}-\overrightarrow{j_{e}} ; \\
\vec{\nabla} \times \vec{H} & =\frac{\partial \vec{E}}{\partial t}+\overrightarrow{j_{g}} ;
\end{aligned}
$$

where $\rho_{e}$ and $\rho_{g}$ are respectively the electric and magnetic charge densities, $\overrightarrow{j_{e}}$ and $\overrightarrow{j_{g}}$ are the corresponding current densities, $\vec{E}$ is electric field and $\vec{H}$ is magnetic field. GDM equations (1) are invariant not only under Lorentz and conformal transformations but also invariant under the following duality transformations $[5,17-20]$,

$$
\begin{array}{ccc}
\vec{E}=\vec{E} & \cos \theta+\vec{H} & \sin \theta ; \\
\vec{H}=-\vec{E} & \sin \theta+\vec{H} & \cos \theta .
\end{array}
$$

For a particular value of $\theta=\frac{\pi}{2}$, equation (2) reduces to

$$
\vec{E} \rightarrow \vec{H} ; \quad \vec{H} \rightarrow-\vec{E},
$$

which can be written as

$$
\left(\begin{array}{l}
\vec{E} \\
\vec{H}
\end{array}\right) \Rightarrow\left(\begin{array}{cc}
0 & 1 \\
-1 & 0
\end{array}\right)\left(\begin{array}{l}
\vec{E} \\
\vec{H}
\end{array}\right)
$$


If we apply the transformation (3) and (4) along with the following duality transformations for current i.e.

$$
\rho_{e} \rightarrow \rho_{g}, \rho_{g} \rightarrow-\rho_{e} \Longleftrightarrow\left(\begin{array}{c}
\rho_{e} \\
\rho_{g}
\end{array}\right) \Rightarrow\left(\begin{array}{cc}
0 & 1 \\
-1 & 0
\end{array}\right)\left(\begin{array}{c}
\rho_{e} \\
\rho_{g}
\end{array}\right)
$$

Differential equation (1) are the generalized field equations of dyons and the corresponding electric and magnetic fields are then called generalized electromagnetic field of dyons are expressed in the following differential form in terms of two potentials [5]

$$
\begin{aligned}
& \vec{E}=-\vec{\nabla} \phi_{e}-\frac{\partial \vec{A}}{\partial t}-\vec{\nabla} \times \vec{B} \\
& \vec{H}=-\vec{\nabla} \phi_{g}-\frac{\partial \vec{B}}{\partial t}+\vec{\nabla} \times \vec{A}
\end{aligned}
$$

where $\left\{A^{\mu}\right\}=\left\{\phi_{e}, \vec{A}\right\}$ and $\left\{B^{\mu}\right\}=\left\{\phi_{g}, \vec{B}\right\}$ are the two four - potentials associated with electric and magnetic charges. Let us define the complex vector field $\vec{\psi}$ in the following form,

$$
\vec{\psi}=\vec{E}-i \vec{H}
$$

equations (6),(7) and (8), thus give rise to the following relation between generalized field and the components of the generalized four - potential as,

$$
\vec{\psi}=-\frac{\partial \vec{V}}{\partial t}-\vec{\nabla} \phi-i \vec{\nabla} \quad \times \vec{V}
$$

Here $\left\{V_{\mu}\right\}$ is the generalized four - potential of dyons and defined as ,

$$
\left\{V_{\mu}\right\}=\{\phi,-\vec{V}\}
$$

where

$$
\phi=\phi_{e}-i \quad \phi_{g}
$$

and

$$
\vec{V}=\vec{A}-i \vec{B}
$$

If we apply the transformation (4) and (5) the following duality transformation for potential is obtained i.e.

$$
\begin{aligned}
& \vec{A} \rightarrow \vec{B}, \vec{B} \rightarrow-\vec{A} \Rightarrow\left(\begin{array}{c}
\vec{A} \\
\vec{B}
\end{array}\right)=\left(\begin{array}{cc}
0 & 1 \\
-1 & 0
\end{array}\right)\left(\begin{array}{l}
\vec{A} \\
\vec{B}
\end{array}\right) \\
& \phi_{e} \rightarrow \phi_{g}, \phi_{e} \rightarrow-\phi_{g} \Rightarrow\left(\begin{array}{c}
\phi_{e} \\
\phi_{g}
\end{array}\right)=\left(\begin{array}{cc}
0 & 1 \\
-1 & 0
\end{array}\right) \quad\left(\begin{array}{c}
\phi_{e} \\
\phi_{g}
\end{array}\right) .
\end{aligned}
$$


Maxwell field equation (1) may then be written in terms of generalized field $\vec{\psi}$ as,

$$
\begin{aligned}
\vec{\nabla} \cdot \vec{\psi} & =\rho \\
\vec{\nabla} \times \vec{\psi} & =-i \vec{J}-i \frac{\partial \vec{\psi}}{\partial t} ;
\end{aligned}
$$

where $\rho$ and $\vec{J}$ are the generalized charge and current source densities of dyons and given by;

$$
\begin{aligned}
\rho & =\rho_{e}-i \rho_{g} \\
\vec{J} & =\overrightarrow{j_{e}}-i \overrightarrow{j_{g}} .
\end{aligned}
$$

Here, we may write the tensorial form of generalized Maxwell Dirac equation of dyons as,

$$
\begin{aligned}
& F_{\mu \nu, \nu}=j_{\mu}^{e} ; \\
& F_{\mu \nu, \nu}^{d}=j_{\mu}^{g} ;
\end{aligned}
$$

where

$$
\left\{j_{\mu}^{e}\right\}=\left\{\rho_{e}, \overrightarrow{j_{e}}\right\} \quad \text { and }\left\{j_{\mu}^{g}\right\}=\left\{\rho_{g}, \overrightarrow{j_{g}}\right\} .
$$

Defining the generalized field tensor of dyons as;

$$
G_{\mu \nu, \nu}=F_{\mu \nu}-i \quad F_{\mu \nu}^{d}
$$

one can directly obtained the following generalized field equation of dyon i.e.

$$
\begin{aligned}
& G_{\mu \nu, \nu}=J_{\mu} ; \\
& G_{\mu \nu, \nu}=0 ;
\end{aligned}
$$

where

$$
\left\{J_{\mu}\right\}=\{\rho,-\vec{J}\} .
$$

The Lorentz four - force equation of motion for dyon is written as;

$$
f_{\mu}=m_{0} \ddot{x}_{\mu}=\operatorname{Re} Q^{*}\left(G_{\mu \nu} u^{\nu}\right)
$$

where ' $R e^{\prime}$ denotes the real part, $\left\{\ddot{x}_{\mu}\right\}$ is the four - acceleration and $\left\{u^{\nu}\right\}$ is the four velocity of the particle and $Q$ is the generalized charge of dyon.

\section{MAGNETOHYDRODYNAMIC EQUATIONS}

Magnetohydrodynamics (MHD) is the mathematical tool for the low frequency interaction between electrically conducting fluids and electromagnetic fields. MHD is the study of the interaction of electromagnetic fields and conducting fluids. The partial differential equations of MHD are derived from Boltzmann's equation where space and time scales are considered larger than all inherent scale lengths such as the Debye length (gyro - radii) of the charged particles. It is, however, more convenient to obtain the MHD equations in a phenomenological way as the electromagnetic extension of the hydrodynamic equations 
of ordinary fluids, where the main approximation is to neglect the displacement current. The MHD equations thus describe the motion of a perfectly conducting fluid interacting with a magnetic field. Hence, we need to combine Maxwell's equations with the equations of gas dynamics and provide equations of MHD. The modeling consists of a coupling between the three equations of continuum fluid mechanics and the Maxwell equations of electromagnetism, respectively $[1,2,21-25]$. Therefore the set of magnetohydrodynamic (MHD) equations consists the following equations

\section{III.1. Continuity equation}

The first equation associated with the continuum fluid is described as the equation of continuity which is written as

$$
\frac{\partial \rho}{\partial t}+\vec{\nabla} \cdot(\rho \vec{u})=0
$$

where $\rho$ is the charge density and $\vec{u}$ is the particle velocity.

\section{III.2. Equation of motion}

The second equation associated with the continuum fluid is described as the standard form for the MHD equation of motion i.e.

$$
\rho \frac{D \vec{u}}{D t}=\vec{J} \times \vec{H}-\triangle \vec{P}
$$

where $\vec{H}$ is the magnetic field intensity, $\rho$ is the electric charge density, $\triangle \vec{P}$ is the change in momentum due to collision and $\frac{D}{D t}$ is the convective derivative described as

$$
\frac{D}{D t}=\frac{\partial}{\partial t}+\vec{u} \cdot \vec{\nabla}
$$

\section{III.3. MHD Ohm's law}

The third equation associated with the continuum fluid is known as the MHD Ohm's law described as

$$
m n_{e}\left[\frac{\partial \vec{u}}{\partial t}+(\vec{u} \cdot \vec{\nabla}) \vec{u}\right]=e n_{e}[\vec{E}+\vec{u} \times \vec{H}]+\triangle \vec{P}_{e}
$$

where $e$ is the electric charge, $m$ is the mass of the particle, $u$ is the particle speed, $n_{e}$ is the number density of electric charge, $\vec{E}$ and $\vec{H}$ are the electromagnetic field intensities $\triangle \vec{P}_{e}$ is the change in momentum due to collision of electrons. 


\section{III.4. Maxwell Equations for electromagnetic fields}

The other differential equations of MHD are associated with following Maxwell's equations

$$
\begin{aligned}
\vec{\nabla} \cdot \vec{E}=\rho_{e} ; \\
\vec{\nabla} \cdot \quad \vec{H}=0 ; \\
\vec{\nabla} \times \vec{E}=-\frac{\partial \vec{H}}{\partial t} \\
\vec{\nabla} \times \vec{H}=\frac{\partial \vec{E}}{\partial t} \quad+\quad \overrightarrow{j_{e}} ;
\end{aligned}
$$

where $\vec{j}$ is the current density due to electron flow.

\section{MAGNETOHYDRODYNAMIC (MHD) EQUATIONS FOR DYONS}

We may now generalize the MHD as the study of the interaction of electromagnetic fields of dyons (particles carrying simultaneous existence of electric and magnetic charges) and conducting fluids carrying electric and magnetic charges. In order to write the MHD equation for dyons, we apply the principle of electromagnetic duality for electric and magnetic counterparts of dyons. For brevity we have used the natural units $c=\hbar=1$ and $\mu_{0}=\varepsilon_{0}=1$. Let us generalize accordingly the Magnetohydrodynamic (MHD) equations for dyons as

\section{IV.1. Generalized MHD continuity equation for dyons}

MHD continuity equation for pure electric charge(in the absence of magnetic monopole) may be written as

$$
\frac{\partial \rho_{e}}{\partial t}+\vec{\nabla} \cdot\left(\rho_{e} \vec{u}\right)=0
$$

Similarly, taking into account the electron-monopole duality, we may write the continuity equation for magnetic monopole as

$$
\frac{\partial \rho_{m}}{\partial t}+\vec{\nabla} \cdot\left(\rho_{m} \vec{u}\right)=0
$$

where $\rho_{m}$ is the magnetic charge density. As such, for the case of plasma of dyon, we may generalize equations (27) and (28) into the following continuity equation

$$
\frac{\partial \rho}{\partial t}+\vec{\nabla} \cdot(\rho \vec{u})=0
$$

where $\rho=\rho_{e}-i \rho_{g}$ is the generalized charge density of dyons described earlier in Section II. 


\section{IV.2. Generalized MHD fluid equation of motion for dyons}

Similarly, we may express the second equation of magnetohydrodynamics (MHD) for the plasma of dyons. Let us start with Boltzmann equation as

$$
\frac{\partial f}{\partial t}+\vec{v} \cdot \vec{\nabla} f+\frac{\vec{F}}{m} \frac{\partial f}{\partial v}=\left(\frac{\partial f}{\partial v}\right)_{C}
$$

where the function $f$ is a velocity distribution function, $F$ is the force acting on the particle. $\left(\frac{\partial f}{\partial v}\right)_{C}$ is the time rate of change of $f$ due to collisions. Here we replace the force $\vec{F}$ of equation (30) as the force exerting on the particle simultaneously carrying electric and magnetic charges (dyons).

$$
\vec{F}=e(\vec{E}+\vec{v} \times \vec{H})+g(\vec{H}-\vec{v} \times \vec{E} \quad) .
$$

Substituting the equation (31) for force $\vec{F}$ of dyons into equation (30) and multiplying by $m \vec{v}$ and then integrating it over the velocity $d \vec{v}$, we get,

$$
\begin{aligned}
m \int \vec{v} \frac{\partial f}{\partial t} d & \vec{v}+m \int \vec{v}(\vec{v} \cdot \vec{\nabla} f) d \vec{v} \\
& +\int \vec{v}[e(\vec{E}+\vec{v} \times \vec{H})+g(\vec{H}-\vec{v} \times \vec{E})] \frac{\partial f}{\partial v} d \vec{v}=\int m \vec{v}\left(\frac{\partial f}{\partial t}\right)_{c} d \vec{v} .
\end{aligned}
$$

The first term of equation (32) reduces to

$$
m \int \vec{v} \frac{\partial f}{\partial t} d \vec{v}=m \frac{\partial}{\partial t} \int \vec{v} f d \vec{v}=m \frac{\partial}{\partial t} \quad(n \vec{u}) .
$$

where $n$ is density function and $\vec{u}$ is the average fluid velocity. Similarly, the second term of equation (32) is expressed as,

$$
m \int \vec{v}(\vec{v} \cdot \vec{\nabla} f) d \vec{v}=m \vec{u} \vec{\nabla} \cdot(n \vec{u})+m n(\vec{u} \cdot \vec{\nabla}) \vec{u}
$$

Thus, the third term of equation (32) is expressed as

$$
e \int \vec{v}(\vec{E}+\vec{v} \times \vec{H}) \frac{\partial f}{\partial v} d \vec{v}=-e n_{e}(\vec{E}+\vec{u} \times \vec{H}) .
$$

Similarly the fourth term of equation (32) reduces to

$$
g \int \vec{v}(\vec{H}-\vec{v} \times \vec{E}) \frac{\partial f}{\partial t} d \vec{v}=-g n_{g}(\vec{H}-\vec{u} \times \vec{E}) .
$$

where $n_{e}$ and $n_{g}$ are respectively the number densities of electric and magnetic charges. On the other hand, the right hand side term of equation (32) is analogous to the change in momentum $\triangle \vec{P}$ due to collision i.e;

$$
\triangle \vec{P}=\int m v\left(\frac{\partial f}{\partial t}\right)_{c} d \vec{v}
$$


Using equations $(33,34,35,36)$ and $(37)$, we get the following reduced form of equation (32) i.e.

$$
\begin{aligned}
m n\left[\left(\frac{\partial \vec{u}}{\partial t}\right)+(\vec{u} \cdot \vec{\nabla}) \vec{u}\right] & =\left(\rho_{e} \vec{E}+\rho_{g} \vec{H}\right)+\left(\overrightarrow{j_{e}} \times \vec{H}-\overrightarrow{j_{g}} \times \vec{E}\right)+\Delta \vec{P} \\
& =\operatorname{Re}\left(\rho \overrightarrow{\psi^{\star}}\right)+\operatorname{Im}\left(\vec{j} \times \overrightarrow{\psi^{\star}}\right)+\triangle \vec{P}
\end{aligned}
$$

which is the MHD equation of motion for the case of dyonic plasma. In equation (38) $\vec{\psi}$ is complex electromagnetic vector field of dyon, $\overrightarrow{\psi^{*}}$ is the complex conjugate of the the dyonic field and $\vec{J}=\overrightarrow{j_{e}}-i \overrightarrow{j_{g}}$ is generalized current source density of dyon. Physical interpretation of equation (38) is thus describes due to the scattering of electric and magnetic charge carriers of dyons. Equation (38) is also described as the fluid equation of motion for generalized field of dyons (i.e. dyonic fluid) which is the modified form of second equation for magnetohydrodynamics (MHD) in case of dyonic plasma.

\section{IV.3. Generalized MHD Ohm's law for dyons}

Third equation of magnetohydrodynamics (MHD) given by equation (25) is recalled as the modified form of Ohm's law. Applying the duality principle, the magnetohydrodynamics (MHD) equation (25) may now be written for pure magnetic charge as

$$
m n_{g}\left[\frac{\partial \vec{u}}{\partial t}+(\vec{u} \cdot \vec{\nabla}) \vec{u}\right]=g n_{g}[\vec{H}-\vec{u} \times \vec{E}]+\triangle P_{g}
$$

Multiplying equation (25) by $\frac{e}{m}$ and equation (39) by $\frac{i g}{m}$ and then subtracting, we get

$$
\left(e n_{e}-i g n_{g}\right)\left[\frac{\partial \vec{u}}{\partial t}+(\vec{u} \cdot \vec{\nabla}) \vec{u}\right]=\frac{e^{2} n_{e}}{m}[\vec{E}+\vec{u} \times \vec{H}]+\frac{e}{m} \triangle \vec{P}_{e}-i \frac{g^{2} n_{g}}{m}[\vec{H}-\vec{u} \times \vec{E}]-i \frac{g}{m} \triangle \overrightarrow{P_{g}} .
$$

Let us define the electric and magnetic charge source densities as

$$
\begin{aligned}
& \overrightarrow{j_{e}}=e n_{e} \vec{u} ; \\
& \overrightarrow{j_{g}}=g n_{g} \vec{u} .
\end{aligned}
$$

Using equations (24) and (41), we get

$$
\frac{D \vec{J}}{D t}=\frac{1}{m}\left[e^{2} n_{e} \vec{F}_{e}-i g^{2} n_{g} \vec{F}_{g}\right]+\frac{1}{m}\left(e \triangle \overrightarrow{P_{e}}-i g \triangle \overrightarrow{P_{g}}\right)
$$

where $\vec{F}_{e}=e(\vec{E}+\vec{u} \times \vec{H})$ is the force due to electric charge and $\overrightarrow{F_{g}}=g(\vec{H}-\vec{u} \times \vec{E})$ is the force due to magnetic monopole. As such, the equation (42) is the modified form of the generalized Ohm's law for magnetohydrodynamics (MHD) associated with the generalized fields of dyons (i.e. the case of dyonic plasma).

\section{IV.4. MHD Maxwell equation for electromagnetic fields for dyons}

Generalized -Dirac-Maxwell (GDM) equations for generalized electromagnetic fields for dyons are already given by equation (1) in Sec. 2. These equations are now be visualized as the another set of MHD equations for generalized fields for dyons. 


\section{FREQUENCY OF DYONIC PLASMA}

The case of dyonic plasma is now be considered as not only the case of single particle motions but rather a collective motion of the various charge species of conductive fluids which contain the electric and magnetic charges both. As such, the dyonic plasma is the generalization of two different kinds of plasmas namely the electric plasma and monopole plasma. So, let us discuss first the electrostatic plasma oscillation responsible for plasma frequency. For this we may start from the electrostatic force

$$
m \overrightarrow{\ddot{r}}=e \vec{E}
$$

where the symbols have their usual meanings and $\vec{r}$ denotes the acceleration. If $\vec{E}=$ $\vec{E} e^{i(\vec{k} \cdot \vec{r}-\omega t)}$ denotes an incident plane wave, the equation (43) reduces to

$$
m \overrightarrow{\dot{r}}=-\frac{e \vec{E}}{i \omega} .
$$

So the electric current source density is expressed as

$$
\overrightarrow{j_{e}}=n_{e} e \overrightarrow{\dot{r}}=-\frac{n_{e} e^{2} \vec{E}}{i m \omega}=\delta_{e} \vec{E}
$$

where

$$
\delta_{e}=-\frac{n_{e} e^{2}}{i m \omega}
$$

Applying the principle of electromagnetic duality, we may write the magnetic current density associated with monopole of charge $g$ moving in a magnetic field, as

$$
\overrightarrow{j_{g}}=\delta_{g} \vec{H}
$$

where

$$
\delta_{g}=-\frac{n_{g} g^{2}}{i m \omega} .
$$

With the help of Maxwell's Dirac equation (1) and using the relations (41), (45- 48), we get the expression for wave number and frequency for generalized fields of dyons as

$$
k^{2}=\omega^{2}-\frac{n_{e}}{m} q q *+\left(\frac{n_{e} e g}{m \omega}\right)^{2}=\omega^{2}-\omega_{p}^{2}
$$

with

$$
\omega_{p}^{2}=\left(\frac{n_{e}}{m} q q *-\left(\frac{n_{e} e g}{m \omega}\right)^{2}\right)
$$

where $q=e-i g$ is the generalized charge of dyon. In equation (50) $\omega_{p}$ is the now be described as the plasma frequency of the dyon, $q^{\star}$ is the complex conjugate of generalized charge $q$ of dyon and $k$ is known as the usual wave number. 


\section{MAGNETOHYDRODYNAMIC (MHD) WAVES FOR DYONS}

Plasma is a complex fluid that support many plasma wave modes. Restoring forces include kinetic pressure and electromagnetic forces [24,25]. Let us discuss the small amplitude waves propagating through a spatially uniform MHD plasma. For this, we take the two cases.

Case I- we take

$$
\vec{E}+\vec{v} \times \vec{H}=\frac{\overrightarrow{j_{e}}}{\sigma_{e}} \simeq 0
$$

which is the Ohm's law for a plasma of very high conductivity $\sigma_{e}$ associated with the electric charge. Similarly for, case -II, we take

$$
\vec{H}-\vec{v} \times \vec{E}=\frac{\overrightarrow{j_{g}}}{\sigma_{g}} \simeq 0
$$

which may also be identified as the Ohm's law with very high conductivity $\sigma_{g}$ associated with the magnetic monopole. Post multiplying vectorially the fourth differential GDM equation (1) by $\vec{H}$ and rearranging the terms, we get,

$$
\overrightarrow{j_{e}} \times \vec{H}=(\vec{\nabla} \times \vec{H}) \times \vec{H}-\frac{\partial \vec{E}}{\partial t} \times \vec{H}
$$

Similarly, post multiplying vectorially the third Maxwell's Dirac equation (1) by $\vec{E}$, we get

$$
\overrightarrow{j_{g}} \times \vec{E}=-(\vec{\nabla} \times \vec{E}) \times \vec{E}-\frac{\partial \vec{H}}{\partial t} \times \vec{E}
$$

By using equations (53) and (54), the fluid equation (38) for the dyons becomes as

$m n\left[\left(\frac{\partial \vec{u}}{\partial t}\right)+(\vec{u} \cdot \vec{\nabla}) \vec{u}\right]=e n_{e} \vec{E}+g n_{g} \vec{H}+(\vec{\nabla} \times \vec{H}) \times \vec{H}+(\vec{\nabla} \times \vec{E}) \times \vec{E}+\triangle \vec{P}+\frac{\partial}{\partial t}(\vec{H} \times \vec{E})$

where $\triangle P=v_{s}^{2} \vec{\nabla} \rho$, and $v_{s}$ is the speed of the dyon. Now,equation (56) becomes as

$$
\begin{aligned}
m n\left[\left(\frac{\partial \vec{u}}{\partial t}\right)+(\vec{u} \cdot \vec{\nabla}) \vec{u}\right]= & e n_{e} \vec{E}+g n_{g} \vec{H}+(\vec{\nabla} \times \vec{H}) \times \vec{H} \\
& +(\vec{\nabla} \times \vec{E}) \times \vec{E}+v_{s}^{2} \vec{\nabla} \rho+\frac{\partial}{\partial t}(\vec{H} \times \vec{E})
\end{aligned}
$$

With the help of equations (51), (52) and GDM equation (1), we get

$$
\begin{aligned}
\frac{\partial \vec{H}}{\partial t} & =\vec{\nabla} \times(\vec{v} \times \vec{H})-\overrightarrow{j_{g}} \\
\frac{\partial \vec{E}}{\partial t} & =\vec{\nabla} \times(\vec{v} \times \vec{E})-\overrightarrow{j_{e}} .
\end{aligned}
$$


The perturbation transformations [26] are now be considered as

$$
\begin{aligned}
\rho & \longmapsto \rho_{0}+\rho_{1} ; \\
H & \longmapsto H_{0}+H_{1} ; \\
E & \longmapsto E_{0}+E_{1} ; \\
v & \longmapsto v_{1} ;
\end{aligned}
$$

where $\rho_{0}$ is the background density of the unperturbed fluid and $v_{0}=0$ (i.e. the fluid is at rest). Using perturbation transformations (58), we get the following reduced expressions for equations (29) and (56) as

$$
\begin{gathered}
\frac{\partial \rho_{1}}{\partial t}+\rho_{0} \vec{\nabla} \cdot \overrightarrow{v_{1}}=0 \\
\frac{\partial^{2} \overrightarrow{v_{1}}}{\partial t^{2}}+v_{s}^{2} \vec{\nabla}\left[-\vec{\nabla} \cdot \overrightarrow{v_{1}}\right]+\frac{H_{0}}{\rho_{0}}\left[\vec{\nabla} \times\left\{\vec{\nabla} \times\left(\overrightarrow{v_{1}} \times \overrightarrow{H_{0}}\right)-\overrightarrow{j_{g}}\right\}\right] \\
+\frac{E_{0}}{\rho_{0}}\left[\vec{\nabla} \times\left\{\vec{\nabla} \times\left(\overrightarrow{v_{1}} \times \overrightarrow{E_{0}}\right)-\overrightarrow{j_{e}}\right\}\right]=0
\end{gathered}
$$

Here we have used

$$
\begin{aligned}
& v_{A}=\frac{H_{0}}{\left(\rho_{0}\right)^{\frac{1}{2}}} ; \quad v_{B}=\frac{E_{0}}{\left(\rho_{0}\right)^{\frac{1}{2}}} ; \\
& v_{1}(\vec{r}, t)=v_{0} \exp i(\vec{k} \cdot \vec{r}-\omega t) ; \\
& \vec{\nabla} \longrightarrow i \vec{k} \text { and } \frac{\partial}{\partial t} \longrightarrow-i \omega .
\end{aligned}
$$

Hence the dispersion relation (59) reduces to the following expression

$$
\begin{aligned}
-\omega^{2} v_{1} & +\left[v_{s}^{2}+v_{A}^{2}+v_{B}^{2}\right]\left(\vec{k} \cdot \overrightarrow{v_{1}}\right) k \\
& +\left(\vec{k} \cdot \overrightarrow{v_{A}}\right)\left[\left(\vec{k} \cdot \overrightarrow{v_{A}}\right) \overrightarrow{v_{1}}-\left(\overrightarrow{v_{A}} \cdot \overrightarrow{v_{1}}\right) \vec{k}-\left(\vec{k} \cdot \overrightarrow{v_{1}}\right) \overrightarrow{v_{A}}\right]-\frac{\overrightarrow{v_{A}} \times\left(\vec{k} \times \overrightarrow{j_{g}}\right)}{\left(\rho_{0}\right)^{\frac{1}{2}}} \\
& +\left(\vec{k} \cdot \overrightarrow{v_{B}}\right)\left[\left(\vec{k} \cdot \overrightarrow{v_{B}}\right) \overrightarrow{v_{1}}-\left(\overrightarrow{v_{B}} \cdot \overrightarrow{v_{1}}\right) \vec{k}-\left(\vec{k} \cdot \overrightarrow{v_{1}}\right) \overrightarrow{v_{B}}\right]-\frac{\overrightarrow{v_{B}} \times\left(\vec{k} \times \overrightarrow{j_{e}}\right)}{\left(\rho_{0}\right)^{\frac{1}{2}}}=0 .
\end{aligned}
$$

It describes the case of a kind of dyonoacoustic wave. Substituting $\vec{k} \perp \overrightarrow{H_{0}}, \vec{k} \perp \overrightarrow{E_{0}}$ and $\overrightarrow{j_{g}}\left\|\overrightarrow{v_{A}}, \overrightarrow{j_{e}}\right\| \overrightarrow{v_{B}}$, we get

$$
\vec{k} \cdot \overrightarrow{v_{A}}=\vec{k} \cdot \overrightarrow{v_{B}}=0
$$

and

$$
\overrightarrow{v_{A}} \times \overrightarrow{j_{g}}=\overrightarrow{v_{B}} \times \overrightarrow{j_{e}}=0 .
$$

As such, we get the following expression for the dispersion relation

$$
-\omega^{2} \overrightarrow{v_{1}}+\left[v_{s}^{2}+v_{A}^{2}+v_{B}^{2}\right]\left(\vec{k} \cdot \overrightarrow{v_{1}}\right) \vec{k}=0 .
$$


The vector nature of the equation $(64)$ requires that the perturbed fluid velocity $\overrightarrow{v_{1}}$ must be parallel to the propagation direction $\vec{k}$ so that $\vec{k} \cdot \overrightarrow{v_{1}}=k v_{1}$. Hence, the wave is longitudinal in nature and its dispersion relation becomes [26]

$$
v_{\phi}=\frac{\omega}{k}=\sqrt{\left(v_{s}^{2}+v_{A}^{2}+v_{B}^{2}\right)} .
$$

As such, the dyon acoustic waves propagates with velocity $\overrightarrow{v_{\phi}}$ in this case. This is recalled as the dyonoacoustic, dyonosonic or simply compressional wave which involves compression and rarefaction generalized electromagnetic waves associated with dyons along with plasma oscillations.

\section{ENERGY OF DYONS}

The energy of the dyonic plasma is related with the dispersive properties of the wave oscillations. Starting from first principle for the electromagnetic energy density and taking into account the specific features of dispersive relations of electromagnetic waves, we may obtain the expression for electromagnetic energy density (namely the Poynting Theorem) [25]. From the third and fourth Maxwell's Dirac equation (1), we obtain

$$
\vec{E} \cdot(\vec{\nabla} \times \vec{H})-\vec{H} \cdot(\vec{\nabla} \times \vec{E})=\vec{E} \cdot \frac{\partial \vec{E}}{\partial t}+\vec{H} \cdot \frac{\partial \vec{H}}{\partial t}+\overrightarrow{j_{e}} \cdot \vec{E}+\overrightarrow{j_{g}} \cdot \vec{H} .
$$

This equation may then be written as the conservation law of energy as

$$
\frac{\partial W}{\partial t}+\vec{\nabla} \cdot \vec{P}=0
$$

where

$$
\vec{P}=\vec{E} \times \vec{H}
$$

is called the Poynting vector. The rate of change of the energy density $\frac{\partial W}{\partial t}$ is then defined as

$$
\frac{\partial W}{\partial t}=\vec{E} \cdot \frac{\partial \vec{E}}{\partial t}+\vec{H} \cdot \frac{\partial \vec{H}}{\partial t}+\overrightarrow{j_{e}} \cdot \vec{E}+\overrightarrow{j_{g}} \cdot \vec{H}
$$

so that we may obtain the energy density by taking time integration as

$$
\begin{aligned}
W(t) & =W_{0}(t)+\int_{t_{0}}^{t} d t\left[\vec{E} \cdot \frac{\partial \vec{E}}{\partial t}+\vec{H} \cdot \frac{\partial \vec{H}}{\partial t}+\overrightarrow{j_{e}} \cdot \vec{E}+\overrightarrow{j_{g}} \cdot \vec{H}\right] \\
& =W_{0}(t)+\left[\frac{E_{0}^{2}+H_{0}^{2}}{2}\right]+\int_{t_{0}}^{t} d t\left(\overrightarrow{j_{g}} \cdot \vec{H}+\overrightarrow{j_{e}} \cdot \vec{E}\right)
\end{aligned}
$$

where $W_{0}(t)$ is the energy density at reference point $t_{0}$. The quantity $\left(\overrightarrow{j_{g}} \cdot \vec{H}+\overrightarrow{j_{e}} \cdot \vec{E}\right)$ is the rate of change of kinetic energy density of the dyon. This can be seen by taking the dot product of force equation which is taking this form with $\vec{v}$

$$
m \vec{v} \cdot \frac{d \vec{v}}{d t}=\vec{v} \cdot\left(\overrightarrow{F_{e}}+\overrightarrow{F_{g}}\right)=\vec{v} \cdot[e(\vec{E}+\vec{v} \times \vec{H})+g(\vec{H}-\vec{v} \times \vec{E})]
$$


which may also be written as

$$
\frac{d}{d t}\left(\frac{1}{2} m v^{2}\right)=e \vec{E} \cdot \vec{v}+g \vec{H} \cdot \vec{v} .
$$

Since this is the rate of change of kinetic energy of a single dyon, the rate of change of the kinetic energy density $T$ for the entire system of dyons is obtained by summing over the energies of the individual dyons i.e.

$$
\frac{d}{d t}(T)=\sum_{i} \int d v f_{i}\left(e_{i} \vec{E} \cdot \vec{v}+g_{i} \vec{H} \cdot \vec{v}\right)=\vec{E} \cdot \overrightarrow{j_{e}}+\vec{H} \cdot \overrightarrow{j_{g}}=\operatorname{Re}\left(\vec{J} \cdot \overrightarrow{\psi^{\star}}\right) .
$$

This shows that positive value of $\left(\vec{E} \cdot \overrightarrow{j_{e}}+\vec{H} \cdot \overrightarrow{j_{m}}\right)$ increases the kinetic energy of dyons whereas the negative value of $\left(\vec{E} \cdot \overrightarrow{j_{e}}+\vec{H} \cdot \overrightarrow{j_{g}}\right)$ decreases the kinetic energy of dyons. The latter situation is possible only if the dyon starts working with a finite initial kinetic energy density. Since $\left(\vec{E} \cdot \overrightarrow{j_{e}}+\vec{H} \cdot \overrightarrow{j_{g}}\right)$ accounts for the changes in the dyon kinetic energy density, $W$ must be the sum of the generalized electromagnetic field density and the particle energy density.

\section{RESULT AND DISCUSSION}

The foregoing analysis describes the magnetohydrodynamics (MHD) for generalized fields of dyons which are the particles carrying simultaneously electric and magnetic charges. This generalized MHD reproduces the usual MHD if we consider the absence of magnetic monopole on dyons. We have also discussed dyonoacoustic wave equation along with plasma oscillations and calculated the energy of dyons. Accordingly the motion of plasma oscillations for two different fluids associated with the electric and magnetic charges or a composite system of dyons has also been discussed. The present formalism may also be useful for describing dyonic string solution transform under $S L(2, Z)$. If the plasma dynamics becomes too fast, resonances occur with the motions of individual particles which invalidate the MHD equations. Furthermore, effects, such as particle inertia and the Hall effect, which are not taken into account in the MHD equations, may play an important role. Since MHD is a single fluid plasma theory, a single dyon fluid approach is justified because the perpendicular motion is dominated by $\vec{E} \times \vec{H}$ drifts. Here the electric field $\vec{E}$ and magnetic field $\vec{H}$ are considered to be symmetrical and dual invariant for dyons while for the dynamics of individual charges these neither symmetrical nor dual invariant. For the case of slow plasma dynamics, the motions of the dyon and ion fluids become sufficiently different as single fluid approach is no longer tenable. This also occurs whenever the diamagnetic velocities, which are quite different for different plasma species, become comparable to the $\vec{E} \times \vec{H}$ velocity . Furthermore, effects such as plasma resistivity, viscosity, and thermal conductivity, which are not taken into account in the MHD equations, become important in this case. There is a different plasma frequency for each species. For $k$ to be real, only those generalized electromagnetic waves are allowed to pass, for which $\omega>\omega_{p}$. At very high frequencies, $\omega=c k$, dyon can not respond fast enough, and plasma effects are negligible. Thus Plasma frequency sets the lower cuts for the frequencies of electromagnetic radiation that can pass through a plasma. The metals 
shine by reflecting most of light in visible range. The visible light can not pass through the metal because the plasma frequency of electrons in metal falls in ultraviolet region. For frequencies in ultra violet region $(U V)$, metals are transparent. The earth's ionosphere reflects radio waves in the same reason. In the present formalism, we have considered a fluid element of dyonic plasma for which the overall charge is taken to be neutral. So, an external electromagnetic field cannot cause motion of a fluid element as a whole, but will sets up currents due to the motion of opposite charges in opposite directions. Due to these currents, an external electromagnetic field exerts a force on the fluid element and changes its direction of motion. Here we have described the motion of plasma oscillations for two different fluids associated with the electric and magnetic charges or a composite system of dyons. This description is an ideal MHD which gives an attractive mathematical structures with well defined conservation laws.

\section{ACKNOWLEDGMENT}

One of us PSB is thankful to Third World Academy of Sciences for awarding him TWAS-UNESCO Associateship. He is also thankful to Professor Tianjun Li and Prof. Yue-Liang Wu for their hospitality at the Institute of Theoretical Physics, Beijing, China.

\section{REFERENCES}

[1] P. A. Davidson, An Introduction to Magnetohydrodynamics, Cambridge University Press, (2001).

[2] D. R. Nicholson, Introduction to Plasma Theory, Wiley New York, (1983).

[3] J. D. Jackson, Classical Electrodynamics, New York: John Wiley \& Sons 2nd ed., (1975).

[4] H. Alfvén, Nature 150 (1942) 405.

[5] P. S. Bisht and O. P. S. Negi, International Journal of Theoretical Physics, 47(2008) 3108.

[6] Jivan Singh, P. S. Bisht and O. P. S. Negi, Comm. Phys. 18 (2008) 27.

[7] Jivan Singh, P. S. Bisht and O. P. S. Negi, Comm. Phys. 17 (2007) 83.

[8] Jivan Singh, P. S. Bisht and O. P. S. Negi, J. Phys. A: Math. And Theor. 40 (2007) 9137.

[9] Jivan Singh, P. S. Bisht and O. P. S. Negi, Comm. Phys. 17 (2007) 129.

[10] Jivan Singh, P. S. Bisht and O. P. S. Negi, J. Phys. A: Math. And Theor. 40 (2007) 11395.

[11] P. Olesen, Dual strings and magnetohydrodynamics, arXiv:hep-th/9509023v6 8 Nov 1995.

[12] Tyan Yeh and Murray Dryer, Astrophysics and Space Science 117 (1985) 165.

[13] Paul J. Dellar, J. Comput. Phys. 172 (2001) 392; DOI: 10.1006/jcph.2001.6815.

[14] Edward Shuryak, "Deconnement, Monopoles and New Phenomena in Heavy Ion Collisions", arXiv:0908.0737v1 [hep-th] 5 Aug 2009.

[15] O. Coceal, W. A. Sabra and S. Thomas, Europhysics Letter 35 (1996) 277.

[16] P. A. M. Dirac, Proc. Royal Society A133 (1931) 60.

[17] N. Cabibbo and E. Ferrari, Nuovo Cimento 23 (1962) 1147.

[18] C. R. Hagen, Phys. Rev. B140 (1965) 804.

[19] D. Zwanzinger, Phys. Rev. D3 (1971) 880.

[20] D. Singleton, Int. J. Theo. Phys. 34 (1995) 37.

[21] H. Goedbloed and S. Poedts, Principles of MagnetoHydrodynamics, Cambridge Univ. Press (2004).

[22] E. N. Parker, Cosmical Magnetic Fields, Clarendon Press Oxford (1979).

[23] E. R. Priest, Solar Magneto-Hydrodynamics, D. Reidel, Dordrecht (1984).

[24] John Howard, Introduction to Plasma Physics, Free Science (2002).

[25] Paul Murray Bellan, Fundamentals of Plasma Physics, Cambridge University Press (2006).

[26] Peter Alan Davidson, An Introduction to Magnetohydrodynamics, Cambridge University Press, Cambridge, England, (May 2001).

Received 20 September 2011. 